\title{
A 'Machine' for Creating Mathematical Concepts in an Abstract Way, Bidecimal Numbers
}

\author{
Rik Verhulst \\ Department of Mathematics, KdG University of Applied Sciences, Antwerp, Belgium
}

Email address:

rikverhulst@yahoo.fr

To cite this article:

Rik Verhulst. A 'Machine' for Creating Mathematical Concepts in an Abstract Way, Bidecimal Numbers. International Journal of Discrete Mathematics. Vol. 6, No. 1, 2021, pp. 15-22. doi: 10.11648/j.dmath.20210601.13

Received: April 7, 2021; Accepted: May 18, 2021; Published: May 26, 2021

\begin{abstract}
In mathematics, the creation and definition of new concepts is the first step in opening up a new field of research. Traditionally this step originated from intuition by a process of observation, analysis and abstraction. This article will show a general method by which most of the common notions of number theory, geometry, topology, etc., can be introduced in one and the same particular way. Therefore, we only need some of the tools of naïve set theory: a set of terms to which we apply an equivalence relation. This equivalence relation induces a partition of the terms with which we can consequently associate new concepts. By using this method's 'machine' in an abstract way on arbitrary sets of terms we can create new notions at will, as we will show in this article, for instance, for bidecimal numbers of different kinds. The fact that we reverse the usual procedure of intuition before abstraction, doesn't mean that we only create esoteric objects without any meaning. On the contrary, their abstract nature precisely provides our imagination with many possibilities for several interpretations in models in which they become useful. So, for example, we can use our bidecimal numbers to define elementary transformations on a cylinder or on a pile of tori.
\end{abstract}

Keywords: Equivalence Relation, Partition, Mapping, Model, Bidecimal Number of the First Kind, Bidecimal Number of the Second Kind

\section{Introduction}

Many mathematical concepts went through a long process before they got their modern abstract definition. For a long time, numbers, points, lines and surfaces, born out of intuition, were tools rather than well described notions. From the moment when they became basic elements on which theories could be built, it was necessary to reflect more deeply on their nature. Although number theory and geometry have been developed continuously through the ages, the abstract view of numbers and points is rather recent. It is a remarkable fact that fruitful knowledge can be brought about from basic concepts that are not clearly specified. One prime example is the history of the calculus.

Since the second half of the nineteenth century, set theory was created for several purposes and after the Bourbaki group of mathematicians in France had reorganized the whole structure of mathematics taking it as a base, natural numbers can nowadays be regarded as cardinals of finite sets and points as formalistic elements in axiomatic relations.
In this article we will show how we can use the notions of equivalence relation, partition and mapping for building a 'machine' that can produce mathematical notions. First, we will give some examples of well-known concepts, such as natural numbers, integer numbers, rational numbers, real numbers, etc. Then we will use this 'machine' for creating totally new concepts in an abstract way, for example bidecimal numbers of different kinds. Then we will give some concrete models that represent these new objects and wherein they become useful. So we will proceed in an opposite way to how concepts in general usually appear: abstraction before intuition!

\section{Equivalence Relations, Partitions and Mappings}

Instead of repeating all the definitions regarding these notions, we illustrate them in the following example. Let $V$ be a set of coloured objects as follows (Figure 1): 


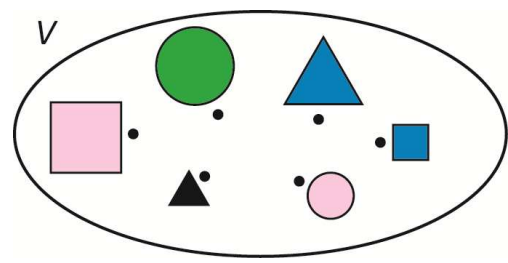

Figure 1. Set of coloured objects.

The human mind can observe properties that some of these objects have in common, e.g.:

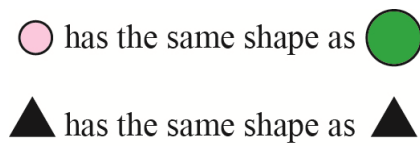

We say that the expression 'has the same shape as' defines a relation $R$ in the set $V$ and we mark the above relations in a short way as:

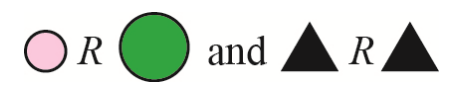

To express these relations in the Venn diagram of the set $V$ we draw an arrow from

$$
\bigcirc \text { to } \bigcirc \text { and a loop from } \triangle \text { to } \Lambda \text {. }
$$
2:

The whole picture of the relation $R$ is then given by figure

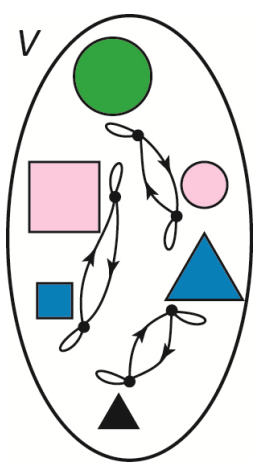

Figure 2. $R$ : 'has the same shape as'.

Since for each object $x$ in $V$ we have $x R x$, we say that $R$ is reflexive.

Since for every two objects $x, y$ of $V$ we have: if $x R y$ then also $y R x$, we say that $R$ is symmetric.

Since for each three objects $x, y, z$ of $V$ we have: if $x R y$ and $y R z$ then also $x R z$, we say that $R$ is transitive.

A relation $R$ that is simultaneously reflexive, symmetric and transitive, is called an equivalence relation. So $R=$ 'has the same shape as 'is an equivalence relation in $V$.

As we can see, the equivalence relation $R$ connects the objects with the same shape in subsets of $V$. All these subsets form a partition of the set $V$. Each object of $V$ belongs precisely to one and only one of these subsets, called equivalence classes.

We can now attribute names or symbols to the equivalence classes and represent them in a new set $M$, e.g. (Figure 3):

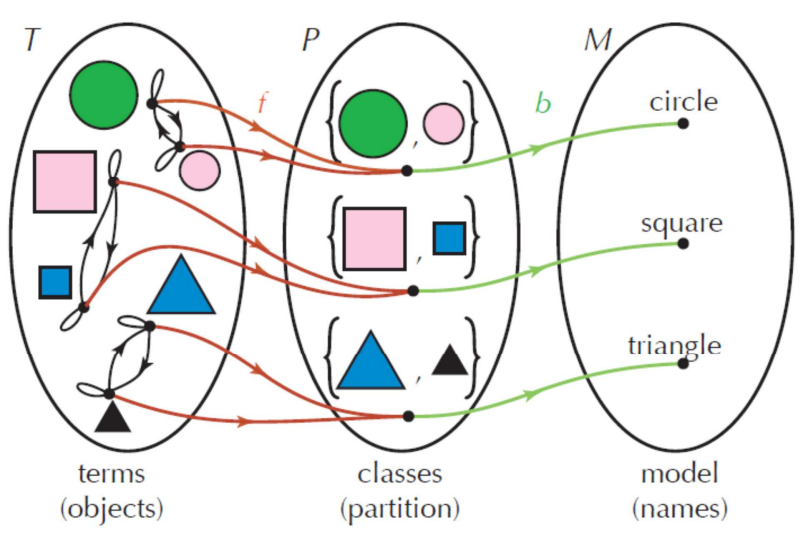

Figure 3. Terms, partition, model.

The set $T=V$ is the set of terms. Equivalent terms are classified in a same subset of $T$. These subsets form the partition $P$ of $T$ (sometimes noticed as $T / R$ ). The names assigned to the equivalence classes of $P$ give a set $M$ of new notions.

The function $f$ is a surjective mapping from $T$ onto $P$. The function $b$ is a bijective mapping from $P$ onto $M$. So the composition $g$ of $f$ and $b$ also is a surjective mapping from $T$ onto $M$.

We now use this three-step 'machine', equivalence relation, partition and mapping, for creating mathematical concepts. All we need is a set of terms and an equivalence relation on these terms. Later on we will show that we can start from a partition of a set as well as from a surjective mapping of a set onto another to define new notions.

\section{Examples of Well-known Concepts}

\subsection{Natural Numbers as Cardinals}

Let us choose as set of terms $T$ a set of finite sets, e.g. the set of non-empty subsets of $\{a, b, c\}$, and let the equivalence relation $R=$ 'have the same number of elements'. Two sets $A$ and $B$ have the same number of elements if and only if there exists a bijection from $A$ onto $B$ (Figure 4).

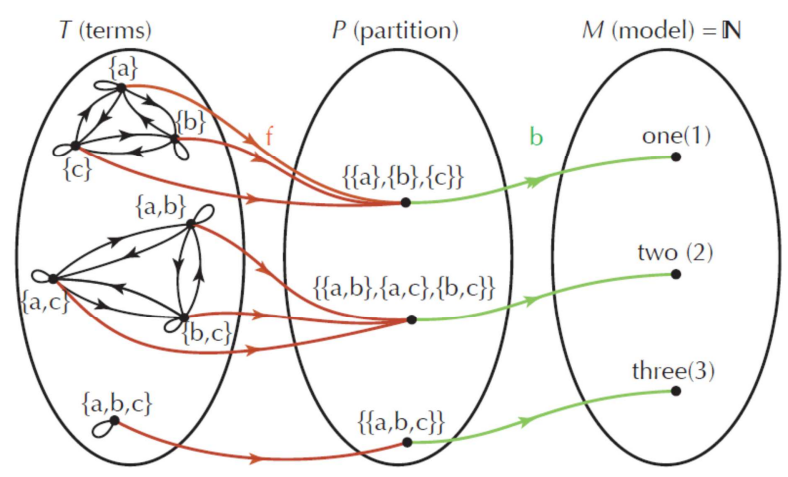

Figure 4. Natural Numbers as Cardinals.

\subsection{Integer Numbers}

Let us choose as set of terms $T$ the product set $\mathbb{N} \times \mathbb{N}=\{(a$, 
b) $\mid a, b \in \mathbb{N}\}$ and let the equivalence relation $R$ be:

$$
(a, b) R(c, d) \Leftrightarrow a+d=b+c .
$$

E.g. $(5,3) R(6,4)$ because $5+4=3+6$ and also $(5,3) R$ $(2,0)$ because $5+0=3+2$

$(4,7) R(1,4)$ because $4+4=7+1$ and also $(4,7) R(0,3)$ because $4+3=7+0$

$(2,2) R(9,9)$ because $2+9=2+9$ and also $(2,2) R(0,0)$ because $2+0=2+0$

It is easy to prove that $R$ is an equivalence relation:

for every $(a, b):(a, b) R(a, b)$ because $a+b=b+a$, so $R$ is reflexive

for every $(a, b)$ and $(c, d):(a, b) R(c, d) \Rightarrow a+d=b+c \Rightarrow c$ $+b=d+a \Rightarrow(c, d) R(a, b)$, so $R$ is symmetric

for every $(a, b),(c, d)$ and $(e, f):(a, b) R(c, d)$ and $(c, d) R(e$, f) $\Rightarrow a+d=b+c$ and $c+f=d+e \Rightarrow a+d+c+f=b+c+d+$ $e=(a+f)+(d+c)=(b+e)+(d+c) \Rightarrow a+f=b+e \Rightarrow(a, b) R$ $(e, f)$, so $R$ is transitive.

Then $(5,3),(6,4)$ and $(2,0)$ are in the same equivalence class; $(4,7),(1,4)$ and $(0,3)$ are in the same equivalence class; $(2,2),(9,9)$ and $(0,0)$ are in the same equivalence class; etc.

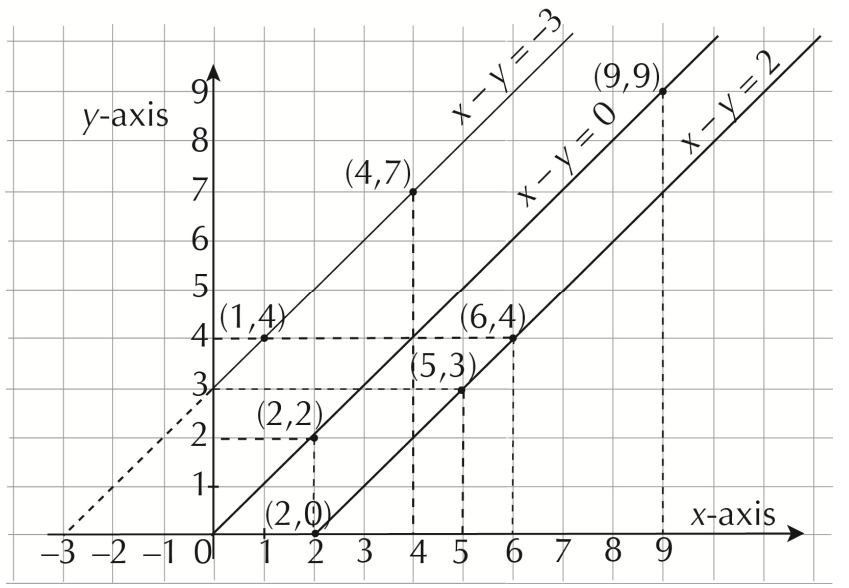

Figure 5. Representation of integers.

We map the class that contains $(2,0)$ onto 2 , the class that contains $(0,3)$ onto -3 , the class that contains $(0,0)$ onto 0 , etc. The set of all the images $M$ under this mapping is the set of the integer numbers $\mathbb{Z}$.

If we represent $\mathbb{N} \times \mathbb{N}$ as points in the first quadrant of a cartesian coordinate system then we can see that the elements of the equivalence class which contains $(x, y)$ are on a line through $(x, y)$ and parallel to the first bisector of the coordinate system (Figure 5). This is obvious, for if $(x, y) R\left(x_{1}, y_{1}\right)$ then $x+y_{1}=y+x_{1}$ and thus $x-y=x_{1}-y_{1}$. This means that $x-y$ is constant for every $(x, y)$ in the same equivalence class. Let $x-y=z$, this is the equation of a line parallel to the first bisector of the cartesian coordinate system. If $y=0$ the element $(x, 0)$ is on the $x$-axis and $x=z$ is the abscissa of the intersection point of the line with equation $x-y=z$ and the line with equation $y=0$ (the $x$-axis). We map that class onto the abscissa $z$ on the $x$-axis.

\subsection{Rational Numbers}

Let us choose as set of terms $T$ the product set $\mathbb{Z} \times \mathbb{Z}_{0}=\{(a$, b) $\mid a, b \in \mathbb{Z}$ and $b \neq 0\}$ and let the equivalence relation $R$ be: $(a, b) R(c, d) \Leftrightarrow a d=b c$.

E.g. $(6,3) R(8,4)$ because $6 \times 4=3 \times 8$ and also $(6,3) R$ $(2,1)$ because $6 \times 1=3 \times 2$

$(2,-7) R(-4,14)$ because $2 \times 14=(-7) \times(-4)$ and also $(2,-7) R(-2,7)$ because $2 \times 7=(-7) \times(-2)$

$(5,5) R(-4,-4)$ because $5 \times(-4)=5 \times(-4)$ and also $(5,5)$ $R(1,1)$ because $5 \times 1=5 \times 1$

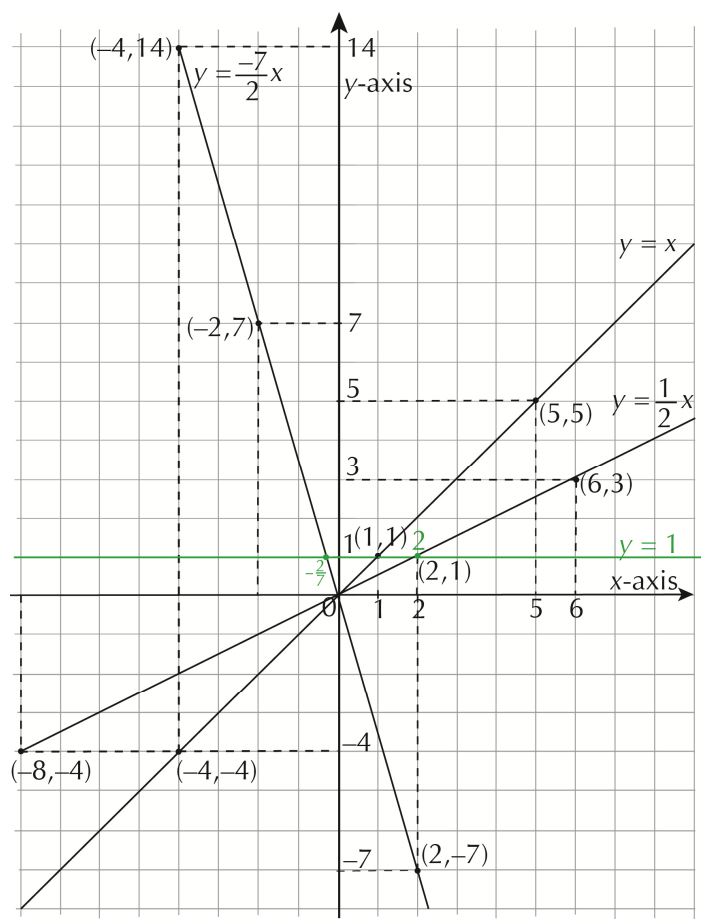

Figure 6. Representation of rational numbers.

We can prove that $R$ is an equivalence relation in the same way as for $R$ in the section on integer numbers, but now using the properties of multiplication instead of addition. Then $(6,3),(8,4)$ and $(2,1)$ are in the same equivalence class; $(2,-7),(-4,14)$ and $(-2,7)$ are in the same equivalence class; $(5,5),(-4,-4)$ and $(1,1)$ are in the same equivalence class; etc. We map the class that contains $(x, y)$ on $\frac{x}{y}$ or $x / y$. In particular $6 / 3=2 / 1=2$ and $2 /(-7)=-2 / 7$. The set $M$ is in this case the set of rational numbers $\mathbb{Q}$.

If we represent $\mathbb{Z} \times \mathbb{Z}_{0}$ as points in a cartesian coordinate system, then we can see that the elements of an equivalence class which contains $(x, y)$ are on a line, different from the $y$ axis, through $(x, y)$ and the origin $(0,0)$ (Figure 6). This is obvious, for if $(x, y) R\left(x_{1}, y_{1}\right)$ then $x y_{1}=y x_{1}$ and thus $x / y=x_{1} / y_{1}$. This means that $x / y$ is constant for every $(x, y)$ in the same equivalence class. Let $x / y=q$ then $y=(1 / q) x$ and this is the equation of a line through the origin $(0,0)$. If $y=1$ the point with coordinate $(x, 1)$ is on the line through the point $(0,1)$ and parallel with the $x$-axis and $x=q=$ the abscissa of the intersection point of the lines with equation $y=(1 / q) x$ and $y=1$. We map that class on the abscissa $q$ on the line with equation $y=1$. 


\subsection{Real Numbers}

In higher education we can choose as set of terms $T$, the set of Cauchy sequences (fundamental sequences) $\mathcal{F}(\mathbb{Q})$ and as equivalence relation $R$ :

$<a_{\mathrm{n}}>R<b_{\mathrm{n}}>\Leftrightarrow<a_{\mathrm{n}}-b_{\mathrm{n}}>$ is a zero-sequence.

A 'Cauchy sequence' is an infinite sequence of rational numbers of which the absolute difference of the progressing terms becomes less than any strict positive rational number. A zero-sequence is a rational sequence that converges to 0 .

With our 'machine' (Figure 7):

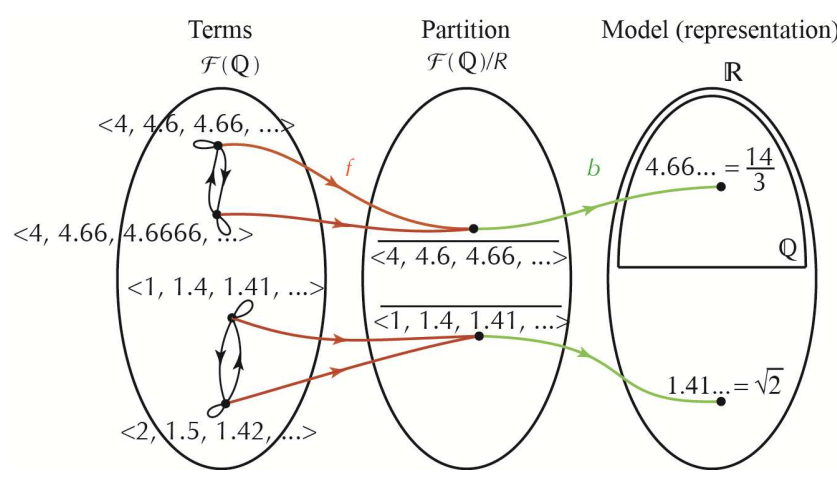

Figure 7. Real numbers as classes of fundamental sequences.

The overscored sequences in $\mathcal{F}(\mathbb{Q}) / R$ represent the equivalence classes of those sequences.

For secondary school pupils we can keep it simpler by choosing as the set of terms the set of decimal expressions, either finite or infinite. E.g. 3.427, 38.33333... or $1.41421356 \ldots$

With such a decimal term, we can define a unique point on a calibrated line. E.g. with $3.427=3.427000 \ldots$ we can associate an infinite sequence of closed intervals: [3, 4], [3.4, 3.5], [3.42, 3.43], [3.427, 3.428], [3.4270, 3.4271], [3.42700, $3.42701], \ldots$ The intersection of this set of intervals is not empty because of the (intuitive) continuity of the line (there are no holes in a straight line). The uniqueness of the number determined by this sequence of closed intervals can be shown by the fact that any other number sooner or later is no longer included in the degressive intervals. That means that the intersection is a singleton. For the example given, the unique point that belongs to every successive interval is $3.427000 \ldots=3.427$.

If we consider the decimal expression $3.426999 \ldots$, the sequence of associated intervals is: [3, 4], [3.4, 3.5], [3.42, $3.43], \quad[3.426, \quad 3.427], \quad[3.4269,3.4270], \quad[3.4299$, $3.42700], \ldots$ The only point that belongs to all these intervals is also $3.427000 \ldots=3.427$.

For this set of terms we define the equivalence relation $R$ as follows:

$x R y \Leftrightarrow x$ defines the same point as $y$

It is trivial that $R$ is an equivalence relation.

So: $3.427000 \ldots R 3.426999 \ldots, 1.8000 \ldots R 1.7999 \ldots$ and $1.7999 \ldots R 1.8$

We prefer to represent each class by one of its decimal terms that does not end in an infinite sequence of zeros or nines. The set $M$ now is the set of real numbers $\mathbb{R}$.

The machine shows (Figure 8):

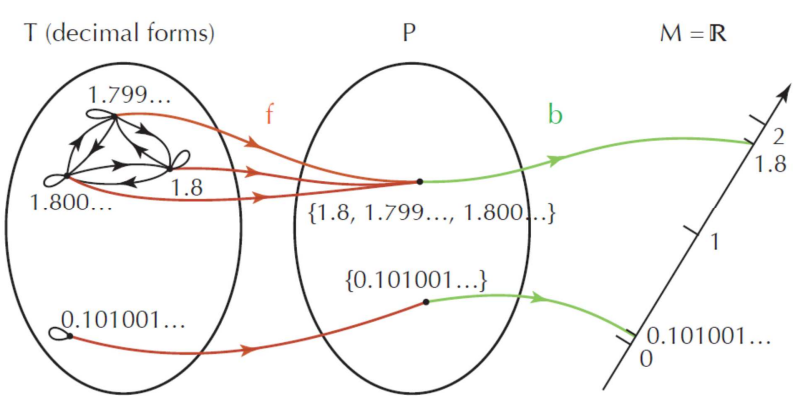

Figure 8. Real numbers as classes of decimal expressions.

\subsection{Real Numbers Modulo 1 (Decapitated Real Numbers)}

Now we choose the real numbers themselves as terms and define another equivalence relation on them in order to obtain a new concept.

So $T=\mathbb{R}$ and $x R y \Leftrightarrow x-y$ is an integer.

E.g. $7.3459 R 2.3459$ because $7.3459-2.3459=5$ and 5 is an integer, $0.666 \ldots R 1.666 \ldots$ because $0.666 \ldots-1,666 \ldots=-1$ and -1 is an integer and $2.157 R-1.843$ because $2.157-(-$ $1.843)=2.157+1.843=4$ and 4 is an integer.

In the case of the negative numbers we can use the logarithmic notation: $-1,843=2+0.157=\overline{2} .157$ Where $\overline{2}=-2$ is the (negative) index and 0.157 the (positive) mantissa.

Then it is clear that using these logarithmic notations we can define the equivalence relation $R$ also as follows: $x R y$ $\Leftrightarrow x$ and $y$ have the same mantissa.

With this definition it is obvious that $R$ is an equivalence relation.

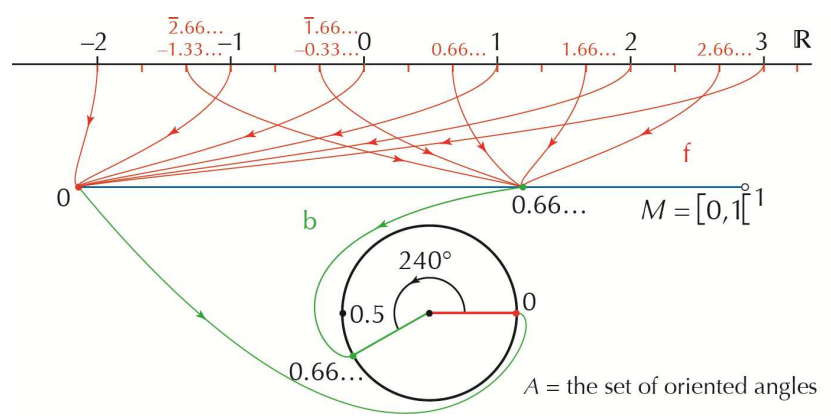

Figure 9. Representation of real numbers modulo 1.

Two equivalent terms differ only by their index. We map the equivalence class of 7.3459 onto its mantissa 0.3459 and the class of $-1.843=\overline{2} .157$ onto its mantissa 0.157 . The set of integer numbers is itself an equivalence class that is mapped onto 0 . The new set $M$ of decapitated real numbers is therefore the half-open interval $[0,1]$. We can curve up this interval to a circle (Figure 9). Every decapitated real number corresponds to just one point on this circle and with respect to a chosen origin on the circle marked by 0 , it also corresponds to a rotation. From that point of view the additional group of decapitated real numbers modulo 1 has many other isomorphic models as different 'costumes'; for example, the composition group of 
rotations around the centre of the circle; the additional group of oriented angles modulo $360^{\circ}$; the multiplicative group of complex numbers with modulus 1 , etc.

With these isomorphisms we can copy each property of the group $[0,1],+$ into the other representations. E.g. each decapitated real number has two halves, because if $r$ is a solution of the equation $2 x=a$, then $r+0.5$ also is a solution, since $2(r+0.5)=2 r+1=a+1=a+0$ (modulo 1$)=a$. So each rotation has two roots for the composition, each oriented angle has two halves modulo $360^{\circ}$ and each complex number modulo 1 has two square roots.

\section{Some Other Examples}

\subsection{Parallel Lines to a Fixed Line A of the Plane}

The set of terms are the points of the plane.

The equivalence relation $R: a R b \Leftrightarrow a$ and $b$ belong to a line parallel to $A$.

The set of equivalence classes is the set of all lines parallel to $A$ (we call it 'the direction of $A$ '), and can be represented by the points of a line not parallel to $A$.

\subsection{Directions of the Plane}

The set of terms is the set of all straight lines of the plane. The equivalence relation $R: A R B \Leftrightarrow A$ is parallel to $B$. The classes are the directions of the plane.

The classes can be represented by the set of all lines through one and the same point.

\subsection{Lengths in the Plane}

The set of terms is the set of all segments of all the lines in the plane.

The equivalence relation $R:[a, b] R[c, d] \Leftrightarrow$ there exists an isometry that maps $[a, b]$ onto $[c, d]$. The classes are all possible lengths of segments in the plane. The classes can be represented by the points on a calibrated line.

\subsection{The Integer Numbers Modulo $n$ Where $n$ Is a Natural Number}

The set of terms are all the integer numbers.

The equivalence relation $R: a R b \Leftrightarrow a$ and $b$ have the same remainder when divided by $n$.

The set of equivalence classes is the set of all the natural numbers less than $n$, i.e. $\mathbb{Z}_{n}$.

\subsection{Starting from a Transformation Group G of a Set V}

The set of terms is (some particular) subsets of $V$

The equivalence relation $R: A R B \Leftrightarrow$ there exists a transformation $f$ in $G$ that maps $A$ onto $B$.

Since in a transformation group we have the identity mapping as a neutral element, $R$ is reflexive.

Since in a transformation group every element has an inverse, $R$ is symmetric.

Since for every two transformations $f$ and $g$ in the group the composition of $f$ and $g$ is also an element of the group, $R$ is transitive.

Hence $R$ is for any transformation group $G$ an equivalence relation. (See example 4.3 as a particular case)

\subsection{Starting from a Subgroup S, * of a Group G, *}

The set of terms is $G$

The equivalence relation $R: a R b \Leftrightarrow$ there exists an element $s$ of $S$ so that $\mathrm{a} * \mathrm{~s}=\mathrm{b}$.

Since $S$ contains the neutral element, $R$ is reflexive.

Since every element of $S$ has an inverse in $S, \mathrm{R}$ is symmetric.

Since for every two elements $s$ and $t$ in $S, s * t$ is also an element of $S, R$ is transitive.

In fact examples 4.1 and 4.4 can be regarded as such.

In example 4.1 we consider the vector group $G,+$ of the plane and as subgroup the group $A_{0},+$ of the line through the origin.

In example 4.4 we consider the group $\mathbb{Z},+$ of the integers and as subgroup the group $n \mathbb{Z},+$ of all the integer multiples of $n$.

\section{New Concepts Starting from a Partition of a Set or from a Mapping onto Another Set}

Our 'machine' has three components: an equivalence relation $R$ in a set of terms; the partition $P$ resulting from this equivalence relation; the mapping onto a representation of the classes of this partition.

In the sections above, we started from the equivalence relation and deduced from that the other two components. We can however start from any of the three components and deduce therefrom the other two.

When we start from a partition $P$ of the set of terms $T$ we can define the equivalence relation $R$ by: $x R y \Leftrightarrow x$ and $y$ belong to the same class of the partition $P$.

When we start from a mapping $g$ of the set of terms onto a representation set $M$ we can define the equivalence relation $R$ by: $x R y \Leftrightarrow x$ and $y$ have the same image under $g$.

So there is no priority among the three steps. No argument anymore between Plato, who claimed the priority of the set $M$ as universal 'ideas', Aristoteles who considered the equivalence relation produced by the observing mind as the first step, and the nominalists who consider the name giving mapping $g$ as essential. Mathematics clears up these different philosophical points of views.

\section{Notions in Daily Life}

In daily life we learn some notions in a similar way. A farmer can distinguish his animals as belonging to different species in three different ways. He can observe the common anatomic properties of horses, cows, pigs, rabbits, etc. and classify them using these resemblances and thus setting up an equivalence relation. Or else he can put them in different stables, which might make it easier for him to keep sight of 
the partition. But the easiest way, which would work for foreigners as well, would be to hang a medallion around their neck with their name on it. The farmer wouldn't give a damn whether he's considered a Platonist, an Aristotelian or a nominalist. Farmers are at times wiser than philosophers.

\section{The Birth of Bidecimal Numbers}

Now we will show how fruitful the 'machine' can be for creating new notions in an abstract way. As an example we will choose as terms 'bidecimal' expressions, finite or infinite.

Examples: 305.41.2879

...431.25.8666...

... 999. $\overline{135} .4700 \ldots$ where $\overline{135}=-135$

In itself such a number is just a superficial appearance without any meaning. To link them to concepts, we have to define an equivalence relation. Depending on our inspiration, we can even do this in different ways. Thus by using the same terms, we can however create several different concepts. We will restrict ourselves to two different versions.

\subsection{Bidecimal Numbers of the First Kind}

\section{Definition 1}

We look at the bidecimal term as being composed of two parts: the part before the first point we consider as a decapitated real number, i.e. as an element of the interval [0, 1], and the part remaining behind the first point as an ordinary real number, i.e. an element of $\mathbb{R}$.

Thus

$\ldots b_{2} b_{1} . z . a_{1} a_{2} \ldots$ is equivalent to $\ldots b_{2}{ }^{\prime} b_{1}{ }^{\prime} . z^{\prime} \cdot a_{1}{ }^{\prime} a_{2}{ }^{\prime} \ldots$

if and only if

$$
0 . b_{1} b_{2} \ldots=0 . b_{1}{ }^{\prime} b_{2}{ }^{\prime} \ldots(\text { in }[0,1])
$$

and $z$. $a_{1} a_{2} \ldots=z$ '. $a_{1}$ ' $a_{2}$ ' ... (in $\mathbb{R}$ )

Hence equivalent bidecimal terms of this first kind will determine equal objects of the first kind.

Examples:

$13.27 .99 \ldots 9 \ldots=13.28 .00 \ldots 0 \ldots$

$\ldots 9 \ldots 99.15 .34=\ldots 0 \ldots 00.15 .34$

$\ldots 9 \ldots 9936 . \overline{13} .99 \ldots 9 \ldots=46 . \overline{12} .00 \ldots 0 \ldots$

We seek a useful representation for these objects. A line segment, closed at the left edge and open at the right, is a good model for the interval $[0,1]$ and a calibrated line is a good model for $\mathbb{R}$. So in first instance we can represent such an object as a point on an infinite strip, closed on the left and open on the right. This infinite strip represents the settheoretical product of $[0,1]$ with $\mathbb{R}$, i.e. $[0,1] \times \mathbb{R}$, resulting in the first model (Figure 10, left).

In the second instance, we can however curve that strip and make the open edge match the closed one, resulting in a cylinder-shaped surface. That gives a second representation (Figure 10, right). Thereby the first component, i.e. a decapitated real number, determines a rotation along a parallel circle. The second component, i.e. a real number, determines a shift parallel to the axis of rotation. The composition of that rotation and that shift we call a screwing, which is just an elementary transformation of that cylinder (mapping between points of the cylinder). Each point on the cylinder then determines, respective to the fixed origin, a screwing which in itself represents a bidecimal of the first kind.
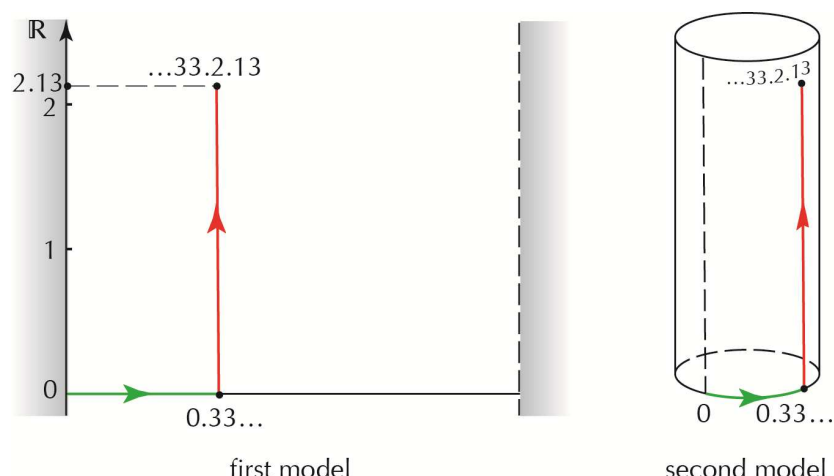

second model

Figure 10. Representation of bidecimal numbers of the first kind.

These bidecimals of the first kind can also be regarded as a representation of the product group of $[0,1],+$ and $\mathbb{R},+$. In this product group we can induce an addition operation, working on the two different components.

When calculating in this group of bidecimals of the first kind, one result is that there are two different halves for each bidecimal.

Example:

$2 a=\ldots 33.2 .13 \Leftrightarrow a=\ldots 661.1 .065$ or $a=\ldots 666.1 .065$

since $2 \cdot 0.166 \ldots=0.33 \ldots$ and $2 \cdot 0.666 \ldots=1.33 \ldots=0.33 \ldots$ (in $[0,1])$

and $2 \cdot 1.065=2.13$ (in $\mathbb{R}$ )

This also implies that each screwing has two different square roots (Figure 11):
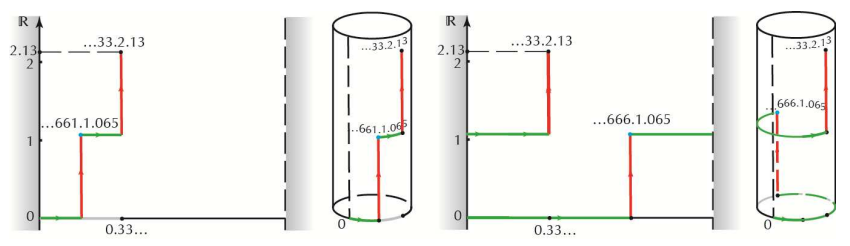

Figure 11. Representation of the two different halves of...33.2.13

\subsection{Bidecimal Numbers of the Second Kind}

\section{Definition 2}

This time we consider the bidecimal terms as consisting of three different components: the part before the first point as a decapitated real number, the part between both points as an integer number, and the part behind the second point again as a decapitated real number.

Thus:... $b_{2} b_{1}$. z. $a_{1} a_{2} \ldots$ is equivalent to... $b_{2}{ }^{\prime} b_{1}{ }^{\prime} . z^{\prime}$. $a_{1}{ }^{\prime} a_{2}{ }^{\prime} \ldots$ if and only if

$$
0 . b_{1} b_{2} \ldots=0 . b_{1}{ }^{\prime} b_{2}{ }^{\prime} \ldots(\text { in }[0,1])
$$

and $\mathrm{z}=\mathrm{z}^{\prime}$ (in $\mathbb{Z}$ )

and $0 . a_{1} a_{2} \ldots=0 . a_{1}{ }^{\prime} a_{2}, \ldots($ in $[0,1])$

Equivalent terms of this second kind determine equal objects of this second kind.

Examples:

$13.27 .99 \ldots 9 \ldots=13.27 .00 \ldots 0 \ldots$ 


\section{$\ldots 9 \ldots 99 . \overline{13} .99 \ldots 9 \ldots=0 . \overline{13} .00 \ldots 0 \ldots$}

We will show some models representing these numbers as well. The integer component can be represented by a point on a calibrated vertical line. The two other components can be represented by a point in a half-open rectangle with these components as sides. A bidecimal number of the second kind then corresponds to a point on a 'floor' of a building that consists of parallel rectangles of which the sides have length 1. E.g. ...33.1.66 ... determines a point on the floor of rank 1 (Figure 12).

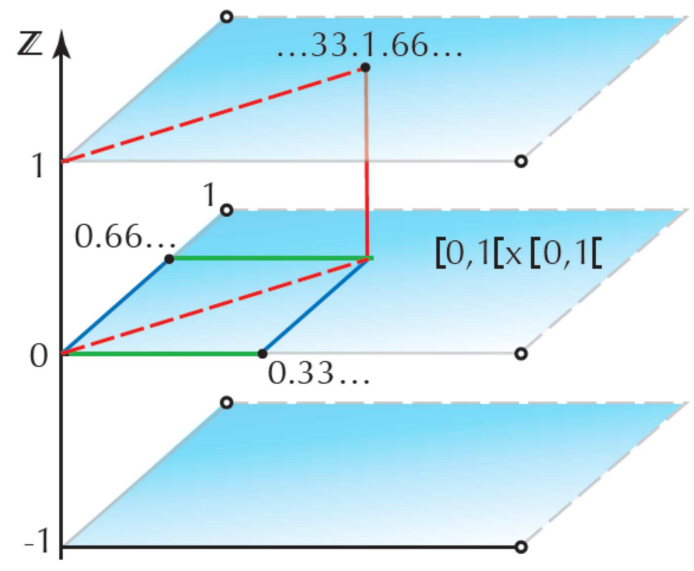

Figure 12. First representation of...33.1.66...

If we curve the rectangle so that one open edge matches the opposite closed edge, the result is a cylinder. If we then make the remaining edges match, we get a torus (like a tube for a vehicle tyre). Then the bidecimals of the second kind are represented by a pile of tori (Figure13). On this pile the first component (decapitated real number) determines a rotation along a parallel circle. The second component (integer number) determines a shift to a floor of the pile. And the third component (decapitated real number) determines a rotation along a meridian circle. The composition of these three mappings is an elementary transformation of the pile of tori (mapping between points of the pile). The order of the mappings in this composition is arbitrary. So we get a second representation:
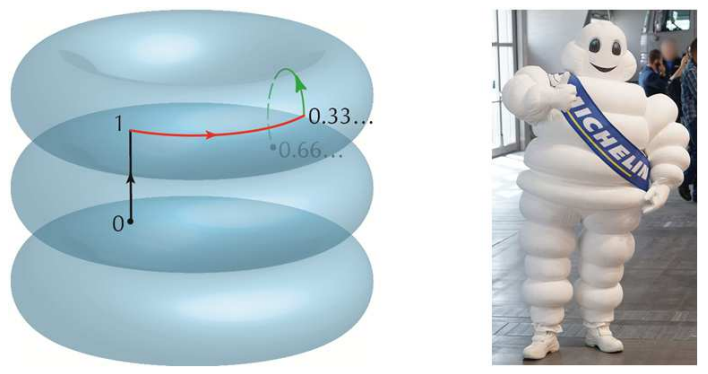

Figure 13. Second representation of...33.1.66...

These bidecimal numbers of the second kind can be regarded as a representation of the product group of three groups: $[0,1],+$ and $[0,1],+$ and $\mathbb{Z},+$. When calculating in this product group by operating on each of the three components we get the astonishing result that a bidecimal number of the second kind with an even integer component has four different halves and one with an odd integer component has none! That also means that an elementary transformation of the torus-building has four square roots when located on a 'floor' of even rank and none if it is located on a 'floor' of odd rank.

$$
\text { Example: } \begin{aligned}
2 x=723.4 .183 \Leftrightarrow & x=5361.2 .0915 \\
& \text { or } x=5366.2 .0915 \\
& \text { or } x=5361.2 .5915 \\
& \text { or } x=5366.2 .5915
\end{aligned}
$$

The half of an odd integer number is not an integer number anymore.

This shows that by using the technique of classification of equivalent terms we can create concepts at will in an abstract way.

The artificial creatures that result can be employed by our imagination in meaningful contexts in which they can be integrated in a useful way. In the course of history lots of mathematical concepts have been created in this way, and often only much later did they get a useful interpretation and application.

\section{Maximal Number of New Concepts Related to a Finite Set}

Because every possible equivalence relation on a set $T$ corresponds to a particular partition of $T$ and vice versa, we can solve the problem of the maximal number of notions related to a set $T$ by counting the number of possible partitions of $T$.

There is a recursive relation between the number of partitions in $i$ classes of a set with $n+1$ elements and the number of partitions of a set with $n$ elements in respectively $i$ -1 and $i$ classes. So we can calculate the number of partitions of a set with $n$ elements starting from a set with one element $\{a\}$, that has only one partition $\{\{a\}\}$, and a set with two elements $\{a, b\}$ that has two partitions, namely $\{\{a, b\}\}$ and $\{\{a\},\{b\}\}$.

If we use the notation $P(i, n)$ for the number of partitions in $i$ classes of a set with $n$ elements then the recursive formula is:

$$
P(i, n+1)=P(i-1, n)+i \cdot P(i, n) .
$$

This formula is easy to prove: Let $T$ be a set with $n$ elements and let $x \notin T$, then we can construct a partition $P$ of $T \cup\{x\}$ in $i$ classes in two steps starting from a partition of $T$ :

a. let $P$ be a partition of $T$ in $i-1$ classes, then $P \cup\{\{x\}\}$ is a partition of $T \cup\{x\}$ in $i$ classes, the number of such partitions is: $P(i-1, n)$

b. let $P$ be a partition of $T$ with $i$ classes then we get a partition of $T \cup\{x\}$ in $i$ classes by adding $x$ to one particular class of $P$ at one time. Since $P$ has $i$ classes, the number of such partitions is: $i \cdot P(i, n)$.

c. So all together, the number of partitions of $T \cup\{x\}$ in $i$ classes is: $P(i-1, n)+i \cdot \mathrm{P}(i, n)$.

With this formula we can set up the table in figure 14: 


\begin{tabular}{|c|c|c|c|c|c|c|c|c|c|c|}
\hline $\mathbf{n i}$ & $\mathbf{1}$ & $\mathbf{2}$ & 3 & 4 & 5 & $\mathbf{6}$ & 7 & $\mathbf{8}$ & $\mathbf{9}$ & $\mathbf{1 0}$ \\
\hline $\mathbf{1}$ & $\mathbf{1}$ & & & & & & & & & \\
\hline $\mathbf{2}$ & 1 & 1 & & & & & & & & \\
\hline 3 & 1 & 3 & 1 & & & & & & & \\
\hline 4 & 1 & 7 & 6 & 1 & & & & & & \\
\hline $\mathbf{5}$ & 1 & 15 & 25 & 10 & 1 & & & & & \\
\hline $\mathbf{6}$ & 1 & 31 & 90 & 65 & 15 & 1 & & & & \\
\hline 7 & 1 & 63 & 301 & 350 & 140 & 21 & 1 & & & \\
\hline $\mathbf{8}$ & 1 & 127 & 966 & 1701 & 1050 & 266 & 28 & 1 & & \\
\hline $\mathbf{9}$ & 1 & 255 & 3025 & 7770 & 6951 & 2646 & 462 & 36 & 1 & \\
\hline $\mathbf{1 0}$ & 1 & 511 & 9330 & 34105 & 42525 & 22827 & 5880 & 750 & 45 & 1 \\
\hline
\end{tabular}

Figure 14. Recursive table for the number of partitions.

E.g. $P(4,7)=P(3,6)+4 \cdot P(4,6)=90+4 \cdot 65=350$

For finding the total number of partitions of a set with $n$ elements we have to add all the terms of the $n$-th row. For example, for the set of the six coloured objects we started with:

$$
1+31+90+65+15+1=203
$$

Instead of using the blocks for building various attractive constructions, a child could make more interesting use of them by partitioning them and finding the 203 hidden new notions. With 8 elements we already get 4140 in number. How impressive the power of mathematical creation is!

\section{Conclusion}

It is always fruitful to ponder methods of an abstract nature. Such methods get creative because they are no longer guided by intuition but by imagination. Mathematicians agree that mathematics is beautiful, at least by itself. But moreover, mathematics has proved its 'unreasonable effectiveness' in many ways. We have many examples in the history of mathematics where abstract concepts were created before becoming useful, whether sooner or later. A wise lesson: don't differentiate between applied mathematics and pure mathematics, but speak about applied mathematics and not-yet-applied mathematics.

\section{Acknowledgements}

I thank Dirk Van Hemeldonck for proofreading, typesetting and his comments, and both Dirk Van Hemeldonck and Ludo Vereecken for making the beautiful drawings.

\section{References}

[1] Bochner S. (1965) The Role of Mathematics in the Rise of Science. Princeton University Press.

[2] Cohen P. (1966) Set Theory and the continuum Hypothesis. W. A. Benjamin.

[3] Cohn P. M. (1974) Algebra, Volume 1. John Wiley \& Sons.

[4] Eves H. (1964) An Introduction to the History of Mathematics. Holt, Rinehart and Winston.

[5] Flegg G. (1993) Numbers. Their history and meaning. Barnes \& Noble Books.

[6] Hardy G. H. (1960) An introduction to the theory of numbers. Oxford University Press.

[7] Kline M. (1972) Mathematical Thought from Ancient to Modern Times. Oxford University Press.

[8] Lang S. (1980) Algebra. Addison-Wesley.

[9] Martin G. E. (1975) The foundations of Geometry and the Non-Euclidian Plane. Springer-Verlag.

[10] Verhulst R. (1982) Begripsvorming in de Wiskunde. Bidecimale getallen. VWNL-CAHIER, Vereniging voor Wisen Natuurkunde Lovanienses, nr. 09.

[11] Verhulst R. (1992) The universe of the coloured arrows, alias the Papygrams. Bulletin de la Société Mathématique de Belgique, Tijdschrift van het Belgisch Wiskundig Genootschap, vol. 4, nr. 2 serie A.

[12] Verhulst R. (2006) In de ban van wiskunde. Het cultuurverschijnsel mathematica in beschaving, kunst, natuur en leven. Garant: 24-42.

[13] Verhulst R. (2019) Im Banne der Mathematik. Die kulturellen Aspekte der Mathematik in Zivilation, Kunst und Natur. Springer Spektrum: 10-28.

[14] Verhulst R. (1982) Nomograms for the calculation of Roots. International Colloquium on Geometry Teaching. ICMI.

[15] Verhulst R. (2020) Recursive formulas for root calculation inspired by geometrical constructions. The teaching of Mathematics Vol. XXIII, 1, 35-50.

[16] Wilder R. (1952) An introduction to the Foundations of Mathematics. John Wiley. 